\title{
90. Activities of the Somatosensory Cortex as well as the Nucleus Centrum Medianum in the Perception of the Splanchnic Information
}

\author{
Miyoshi Urabe, Takashi Tsubokawa, Yoh Watanabe, Noboru Hamabe, \\ Shuji Asano, Haruhide Ito and Masaki KIDANI \\ Dept. of Surgery, School of Medicine, University of Kanazawa
}

We have already reported that the splanchnically driven unitary discharge (DU) from the CM neuron, which played an important role in the perception of the viscerosensory information, were inhibited by the extensive strychnization of the cerebral cortex, while spontaneous unitary discharge (SU) were facilitated. On the other hand, an entire decortication showed just reverse effect. The present report was about the effects of the stimulation of more localized regions of the cortex, such as $S_{I}$ and $S_{\mathrm{II}}$.

Effect on DU of the CM neuron.

1. Either single shock stimulation or repetitive stimulation with $10 \mathrm{cps}$ of the $S_{I}$ exhibited no facilitation of DU in any neuron, inhibition of DU in $43.5 \%$ of the $\mathrm{CM}$ neuron and caused no effect on DU in $56.5 \%$ of the $\mathrm{CM}$ neuron.

2. Single shock stimulation of the $\mathrm{S}_{\mathrm{II}}$ exhibited a facilitation of DU in $66.8 \%$ of the $\mathrm{CM}$ neuron, an inhibition of DU in $14.3 \%$ of the $\mathrm{CM}$ neuron and caused no effect on DU i n $18.9 \%$ of the neuron. The repetitive stimulation of $S_{I I}$ with 10 cps exhibited a facilitatin of DU in $25.0 \%$ of the $\mathrm{CM}$ neuron an inhibition of DU in $16.7 \%$ of the $\mathrm{CM}$ neuron and no effect on DU in $58.3 \%$ of the CM neuron.

Eflect on $\mathrm{SU}$ of the $\mathrm{CM}$ neuron.

1. Stimulation of the $\mathrm{S}_{\mathrm{I}}$ exhibited a facilitation of SU in $23.0 \%$ of the $\mathrm{CM}$ neuron, an inhibitin of SU in $53.8 \%$ of the $\mathrm{CM}$ neuron caused no effect on SU in $7.7 \%$ of the $\mathrm{CM}$ neuron.

2. Stimulation of the $\mathrm{S}_{\mathrm{II}}$ exhibited a facilitation of $\mathrm{SU}$ in $16.7 \%$ of the $\mathrm{CM}$ neuron, an inhibition of SU in $16.7 \% \mathrm{CM}$ neuron and no effect on $\mathrm{SU}$ in $50 \%$ of the $\mathrm{CM}$ neuron. In the remaining neurons, $\mathrm{SU}$ developed groupingly after cessation of stimuli. 